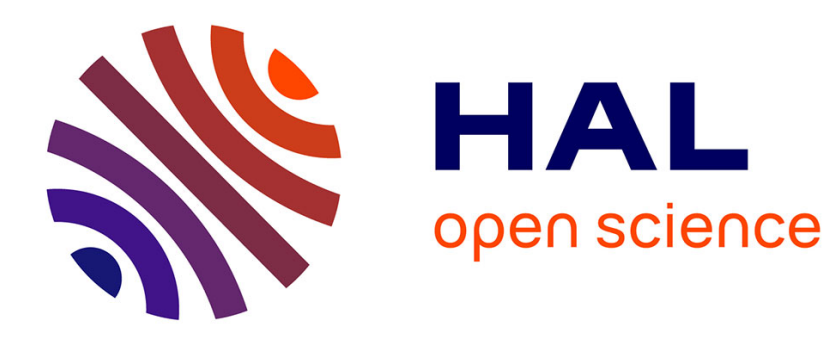

\title{
Nouvelle méthode de détermination des potentiels de sortie des métaux
}

\author{
R. Bedos
}

\section{To cite this version:}

R. Bedos. Nouvelle méthode de détermination des potentiels de sortie des métaux. Revue de Physique Appliquée, 1968, 3 (4), pp.395-399. 10.1051/rphysap:0196800304039501 • jpa-00242877

\section{HAL Id: jpa-00242877 https://hal.science/jpa-00242877}

Submitted on 1 Jan 1968

HAL is a multi-disciplinary open access archive for the deposit and dissemination of scientific research documents, whether they are published or not. The documents may come from teaching and research institutions in France or abroad, or from public or private research centers.
L'archive ouverte pluridisciplinaire HAL, est destinée au dépôt et à la diffusion de documents scientifiques de niveau recherche, publiés ou non, émanant des établissements d'enseignement et de recherche français ou étrangers, des laboratoires publics ou privés. 


\title{
NOUVELLE MÉTHODE DE DÉTERMINATION DES POTENTIELS DE SORTIE DES MÉTAUX
}

\author{
Par R. BEDOS, \\ Laboratoire de Physique Expérimentale, Faculté des Sciences de Marseille, place Victor-Hugo, 13-Marseille ( $\left.3^{\mathrm{e}}\right)$. \\ (Reçu le 21 juin 1968, révisé le 19 septembre.)
}

Résumé. - La méthode proposée est basée sur la théorie de Fowler. C'est une méthode numérique qui permet d'obtenir simultanément, pour un métal donné soumis à l'action d'un agent physique, le potentiel de sortie, sa variation et la variation concomitante du coefficient de photo-ionisation.

Elle diffère des méthodes antérieures basées sur la même théorie par les caractéristiques suivantes :

Elle s'affranchit totalement de la mesure en vraie valeur des flux et des courants qui n'interviennent plus que sous forme de rapport.

Elle ne comporte aucune hypothèse simplificatrice qui limiterait ses possibilités réelles d'utilisation.

Sa mise en œuvre n'introduit pas d'erreurs supplémentaires dues à la méthode elle-même.

En définitive, cette nouvelle méthode est particulièrement adaptée à la détermination des grandeurs caractéristiques de l'effet photoélectrique des métaux.

\begin{abstract}
The proposed method is based on Fowler's theory. It is a numerical method allowing simultaneously determination, for a given metal submitted to the action of a physical agent, of the work function, its variation and the accompanying variation of the photoionisation coefficient.

It differs from earlier methods based on the same theory by the following characteristics :

It becomes totally independent of the measurement expressed in the real value of radiant powers and of intensities which intervene only in the form of a ratio.

It requires no simplifying hypothesis, limiting its real possibilities of application.

Its application does not introduce supplementary errors due to the method itself.

To conclude this new method is particularly adapted for the determination of the characteristic quantities of the photoelectric effect of metals.
\end{abstract}

On sait que la théorie de Fowler [1] constitue une bonne représentation de l'effet photoélectrique des métaux au voisinage du seuil et qu'elle fournit, selon du Bridge [2], pour la sensibilité spectrale (ou rendement) de la cathode, l'expression :

$$
\rho=A \alpha T^{2} f(\delta)
$$

où $A$ est la constante de Dushman, $\alpha$ la probabilité de photo-ionisation, indépendante en principe de $\nu$ dans le domaine de fréquences envisagées et $f(\delta)$ une fonction croissante de la variable :

$$
\delta=(h \nu-\mathrm{e} \varphi) / k T .
$$


Par l'intermédiaire de $\delta$, le rendement photoélectrique est donc à la fois fonction de la fréquence $\nu$, de la température $T$ et du potentiel de sortie $\varphi$; si ce dernier est lui-même fonction d'un paramètre $P, \varphi(P)$, représentant un agent physique quelconque, on peut écrire, pour un métal donné, le rendement sous la forme :

$$
\rho=g(\nu, T, P) \text {. }
$$

Il en serait de même si le coefficient de photo-ionisation dépendait du paramètre $P$.

On dispose de deux procédés pour conduire l'étude de l'effet photoélectrique. On peut dans une première méthode mesurer le rendement en faisant varier la fréquence des radiations incidentes, laissant la température constante ainsi que le paramètre $P$, on est alors conduit à tracer la famille des courbes isothermes :

$$
\rho=g(\nu)_{T, P} \text {. }
$$

Dans une seconde méthode, on mesure le rendement en faisant varier la température $T$ ou le paramètre $P$ en laissant constante la fréquence. On tracera dans ces conditions l'une ou l'autre des familles de courbes isochromatiques :

$$
\rho=g[T]_{\nu, P} ; \quad \rho=g[P]_{\nu, T} .
$$

Toutes les méthodes qui ont été proposées pour déterminer à partir de l'effet photoélectrique les grandeurs caractéristiques du métal étudié (seuil et coefficient de photo-ionisation) entrent dans l'une ou l'autre de ces deux catégories.

Méthode des isothermes. - Le procédé le plus classique a été indiqué par Fowler [1] et Lessen [3] en a donné une transcription numérique, qui peut en améliorer la précision.

Ces méthodes fournissent les valeurs du potentiel de sortie et du coefficient de photo-ionisation correspondant aux valeurs choisies, pendant la durée de l'expérience, pour la température et pour le paramètre. Elles permettent donc en principe l'étude de la variation du potentiel de sortie par exemple, en fonction soit de la température de la cathode, soit de l'action d'un agent physique extérieur. On ne doit pas oublier toutefois qu'elles font nécessairement intervenir la mesure des flux incidents en unité énergétique; d'autre part, dans la méthode graphique de Fowler, la superposition des courbes expérimentale et théorique comporte une indétermination non négligeable. En définitive, on ne peut obtenir avec la méthode des isothermes qu'une précision de quelques pour cent.

Méthode des isochromatiques. - La première application de cette méthode a été proposée par Du Bridge [4]. La fréquence de la radiation incidente est maintenue constante tandis que l'on fait varier la température de la cathode. On compare la courbe théorique $\log f(\delta)$ tracée en fonction de $\log \delta$ à la courbe expérimentale $\log \rho$ tracée en fonction de $\log 1 / T$, la translation qu'il est nécessaire d'effectuer suivant l'axe des abscisses pour amener ces deux courbes en coïncidence dépend du potentiel de sortie. Compte tenu de la variation faible mais certaine du potentiel de sortie avec la température, la valeur ainsi trouvée constitue une pseudo-moyenne de cette grandeur rapportée à l'intervalle de température considéré. On ne peut envisager d'utiliser ce procédé pour étudier l'effet de la température sur le potentiel de sortie; il en est de même avec la méthode de la droite de Richardson pour l'effet thermoélectronique.

Méthode proposée. - L'étude de l'effet photoélectrique des métaux et plus particulièrement le désir de mettre en évidence de faibles variations du potentiel de sortie en fonction d'un facteur physique nous ont amenés à mettre au point une méthode de calcul présentant les caractéristiques suivantes :

- elle ne fait pas intervenir la mesure en vraie valeur des courants de l'émetteur ni celle des flux incidents en unité énergétique;

- elle ne comporte aucune hypothèse simplificatrice, avantageuse sans doute pour résoudre un problème particulier, mais qui limiterait ses possibilités réelles d'utilisation;

- les déterminations auxquelles elle conduit correspondent aux valeurs prises, pendant la durée de l'expérience, par les deux paramètres maintenus constants : température et facteur physique agissant sur l'émetteur;

- l'étude étant conduite en fonction de la fréquence des radiations excitatrices, la méthode proposée s'apparente à la méthode des isothermes, mais se libère des mesures de flux en unité énergétique.

Ceci posé, envisageons l'action d'un agent physique sur l'effet photoélectrique d'un métal; nous supposerons que cette action se manifeste aussi bien sur le potentiel de sortie que sur le coefficient de photoionisation.

Imaginons dans ces conditions une première expérience dans laquelle l'agent physique ayant la valeur $P$ le potentiel de sortie prend la valeur $\varphi$, le coefficient de photo-ionisation la valeur $\alpha$, etc., et une deuxième expérience dans laquelle ces grandeurs deviennent respectivement $P^{\prime}, \varphi^{\prime}, \alpha^{\prime}$, etc., les accroissements correspondants $\Delta P, \Delta \varphi, \Delta \alpha \ldots$, pouvant être considérés comme faibles.

Les mesures effectuées au cours de ces deux expériences conduisent à deux séries de $n$ relations analogues à (1) :

$$
\begin{gathered}
\rho_{i}=\left(\frac{I}{F}\right)_{i}=A \alpha T^{2} f\left(\delta_{i}\right) \\
\rho_{i}^{\prime}=\left(\frac{I^{\prime}}{F^{\prime}}\right)_{i}=A \alpha^{\prime} T^{\prime 2} f\left(\delta_{i}^{\prime}\right) .
\end{gathered}
$$

Chacune des relations de l'une et l'autre série correspond à une valeur déterminée $\nu_{i}$ de la fréquence excitatrice, l'indice $i$ variant de 1 à $n$. 
Divisant membre à membre (4) par (3), il vient :

$$
R_{i}=\left(\frac{I^{\prime}}{F^{\prime}} \cdot \frac{F}{I}\right)_{i}=\frac{\alpha^{\prime}}{\alpha}\left(\frac{T^{\prime}}{T}\right)^{2} \frac{f\left(\delta_{i}^{\prime}\right)}{f\left(\delta_{i}\right)}
$$

en remarquant que :

$$
\delta_{i}=\frac{h \nu_{i}-e \varphi}{k T} ; \quad \delta_{i}^{\prime}=\frac{T}{T^{\prime}} \frac{\left(h \nu_{i}-e \varphi^{\prime}\right)}{k T}
$$

et en posant pour simplifier l'écriture :

$G_{i}=h v_{i} / k T, \quad X=\alpha^{\prime} / \alpha, \quad Y=e \varphi / k T, \quad Z=e \varphi^{\prime} / k T$

il vient :

$$
R_{i}=X \cdot\left(\frac{T^{\prime}}{T}\right)^{2} \cdot \frac{f\left[\frac{T}{T^{\prime}}\left(C_{i}-Z\right)\right]}{f\left[C_{i}-Y\right]}
$$

ou encore :

$$
R_{i}=G\left(C_{i}, X, Y, Z\right)
$$

Nous disposons, pour effectuer le calcul des trois grandeurs inconnues $X, Y, Z$, d'un système de $n$ équations :

$$
\begin{aligned}
& R_{1}=G_{1}\left(G_{1}, X, Y, Z\right) \\
& R_{i}=G_{i}\left(G_{i}, X, Y, Z\right) \\
& R_{n}=G_{n}\left(G_{n}, X, Y, Z\right) .
\end{aligned}
$$

Si les déterminations des $R_{i}$ étaient exactes, ce calcul ne présenterait aucune difficulté car les équations du système (6) seraient compatibles bien qu'en nombre supérieur à celui des inconnues.

Dans la réalité, les valeurs des $R_{i}$ ne sont qu'approchées (elles résultent de mesures expérimentales), on est amené à affecter chacun des $R_{i}$ d'une correction $\Delta R_{i}$ pour obtenir à partir du système (6) le système compatible :

$$
R_{i}+\Delta R_{i}=G_{i} .
$$

Les valeurs les plus probables des corrections $\Delta R_{i}$ et donc des inconnues $X, Y, Z$ sont obtenues en écrivant les trois équations :

$$
\frac{\partial M}{\partial X}=0 ; \quad \frac{\partial M}{\partial Y}=0 ; \quad \frac{\partial M}{\partial Z}=0
$$

avec :

$$
M=\sum_{1}^{n}\left(G_{i}-R_{i}\right)^{2} .
$$

Les expressions (7) constituent le système des trois équations à trois inconnues permettant, théoriquement, de calculer $X, Y, Z$. En pratique, ce calcul n'est possible que si les inconnues entrent sous forme linéaire dans la fonction $G$, ce qui n'est pas ici le cas.

Il est toutefois possible de se ramener à la résolution d'un système linéaire de la façon suivante :

Soient $X_{0}, Y_{0}, Z_{0}$ trois valeurs approchées des quantités cherchées obtenues en résolvant trois des $n$ équations du système (6). On suppose que ces valeurs approchées diffèrent peu des vraies valeurs. On posera donc $X=X_{0}+\Delta X$, etc., les quantités $\Delta X, \Delta Y, \Delta Z$ ainsi introduites étant suffisamment petites pour que l'on puisse se borner aux termes du premier ordre dans le développement de Taylor de la fonction $G$ au voisinage de $X_{0}, Y_{0}, Z_{0}$.

En portant dans le système (7) cette nouvelle forme prise par la fonction $G_{i}$, on obtient finalement le système linéaire cherché :

$$
\begin{aligned}
\frac{1}{2} \frac{\partial M}{\partial X}=\sum_{i} & \frac{\partial G_{i}}{\partial X}\left[\frac{\partial G_{i}}{\partial X} \Delta X+\frac{\partial G_{i}}{\partial Y} \Delta Y\right. \\
& \left.+\frac{\partial G_{i}}{\partial Z} \Delta Z+G_{i}\left(C_{i}, X_{0}, Y_{0}, Z_{\mathbf{0}}\right)-R_{i}\right]
\end{aligned}
$$

et les expressions analogues en $Y$ et $Z$.

La résolution du système linéaire fournit les corrections $\Delta X, \Delta Y, \Delta Z$ à apporter aux valeurs approchées introduites initialement; le processus est ensuite répété en prenant les valeurs corrigées comme valeurs initiales.

En reportant dans le système (6) les valeurs corrigées, on calcule la variance de la fonction $R$, à partir de laquelle on déduit à l'aide du système (8) la variance de chacune des inconnues. Ce calcul est répété à chaque itération jusqu'à obtenir le minimum de ces variances. Généralement trois ou quatre itérations sont suffisantes. La connaissance des variances permet en outre d'estimer de façon statistique la précision des résultats obtenus [5].

Précision de la méthode. - Nous avons déjà indiqué les différents facteurs qui, dans les méthodes classiques, limitent la précision des résultats.

Dans la méthode proposée, le calcul des rapports $R=\frac{I^{\prime}}{I} \cdot \frac{F}{F^{\prime}}$ conduit à comparer entre elles des quantités de même espèce (courants ou flux monochromatiques) qui ont par hypothèse le même ordre de grandeur.

G'est une opération qui, toutes choses égales par ailleurs, est effectuée avec une précision beaucoup plus grande que la détermination de ces mêmes quantités en vraies valeurs comme dans les méthodes classiques.

L'appareil de mesure devra être fidèle et posséder une réponse linéaire, cette dernière condition étant facilement remplie, lorsque l'on opère, comme c'est le cas, au voisinage d'une valeur déterminée.

On peut considérer qu'avec cette méthode le courant de l'émetteur obtenu dans la seconde expérience est mesuré en prenant comme unité le courant obtenu dans la première expérience, il en est de même pour le flux.

Dans ces conditions, le récepteur destiné à mesurer les flux incidents n'a pas besoin d'être étalonné, on peut choisir un appareil sensible (cellule ou photomultiplicateur) auquel on ne demandera que d'avoir une réponse linéaire dans un faible domaine autour du flux contrôlé. 
On comprend dès lors qu'il soit possible d'obtenir avec la méthode proposée une précision supérieure à celle des méthodes classiques.

Exemple d'application. - L'exemple choisi est relatif à l'action de la température sur l'effet photoélectrique du baryum, les calculs ont été effectués à partir des mesures de Jamison et Cashman [6].

Utilisant la méthode graphique de Fowler, ces auteurs avaient obtenu :

pour $T=295,4 \mathrm{o}$ :

$\varphi=2,5106 \mathrm{~V} \quad$ (moyenne de cinq déterminations)

pour $\quad T^{\prime}=397,2 \circ \mathrm{K}$ :

$\varphi^{\prime}=2,5128 \mathrm{~V}$ (moyenne de trois déterminations).

Des indications fournies, il semble que l'on puisse situer la précision de leurs mesures entre 1 et $2 \%$.

Ils ont d'ailleurs fait remarquer que leurs résultats n'étaient pas donnés à $0,0001 \mathrm{~V}$ près, mais que le cinquième chiffre avait été retenu pour servir de terme de comparaison. Si l'on admet la validité du quatrième chiffre, il en résulte un coefficient de température, $a$, positif compris, d'après les auteurs, entre 2,9 et $1,5 \times 10^{-5} \mathrm{~V} /{ }^{\circ} \mathrm{K}$; Meessen [7] a trouvé, à partir de ces mesures, deux valeurs pour ce même coefficient selon qu'il était calculé à 300 ou 400 oK.

$$
\begin{aligned}
& a_{300}=7,3 \times 10^{-5} \mathrm{~V} / \mathrm{o} \mathrm{K} \\
& a_{400}=8,5 \times 10^{-5} \mathrm{~V} / \mathrm{oK} .
\end{aligned}
$$

Aucune mention n'est faite du coefficient de photoionisation.

Nous avons représenté (fig. 1) les données expérimentales sous la forme $R(h v)$. Les calculs nous ont fourni les résultats suivants :

$$
\begin{gathered}
T=295,4 \circ \mathrm{K} ; \quad \varphi=2,523_{5} \pm 0,012 \mathrm{~V} \\
T^{\prime}=397,2 \circ \mathrm{oK} ; \varphi^{\prime}=2,525_{1} \pm 0,016 \mathrm{~V} \\
\alpha^{\prime} / \alpha=0,906 \pm 0,039 .
\end{gathered}
$$

Rappelons que les incertitudes mentionnées ne dépendent pas de la méthode de calcul, mais de la précision des mesures. Compte tenu des erreurs expérimentales, nos valeurs recoupent celles des auteurs.

En toute rigueur, on devrait donc en déduire qu'entre 300 et $400{ }^{\circ} \mathrm{K}$ la précision des mesures ne permet pas de conclure à un effet de la température sur le potentiel de sortie; par contre, la température produit une diminution de $10 \%$ du coefficient de photo-ionisation.

Si l'on accepte toutefois comme une indication les valeurs numériques obtenues pour le potentiel de sortie, on en déduit un coefficient de température de $1,6 \times 10^{-5} \mathrm{~V} /{ }^{\circ} \mathrm{K}$. Une épreuve simple permet de vérifier la qualité de ces déterminations. A partir des valeurs trouvées pour $\varphi$ et $\varphi^{\prime}$, on calcule les fonctions de Fowler $f(\delta)$ et $f\left(\delta^{\prime}\right)$ et l'on forme ensuite, pour chacune des valeurs de la fréquence, la relation :

$$
\frac{\alpha^{\prime}}{\alpha}=R\left(\frac{T}{T^{\prime}}\right)^{2} \frac{f(\delta)}{f\left(\delta^{\prime}\right)} \text {. }
$$

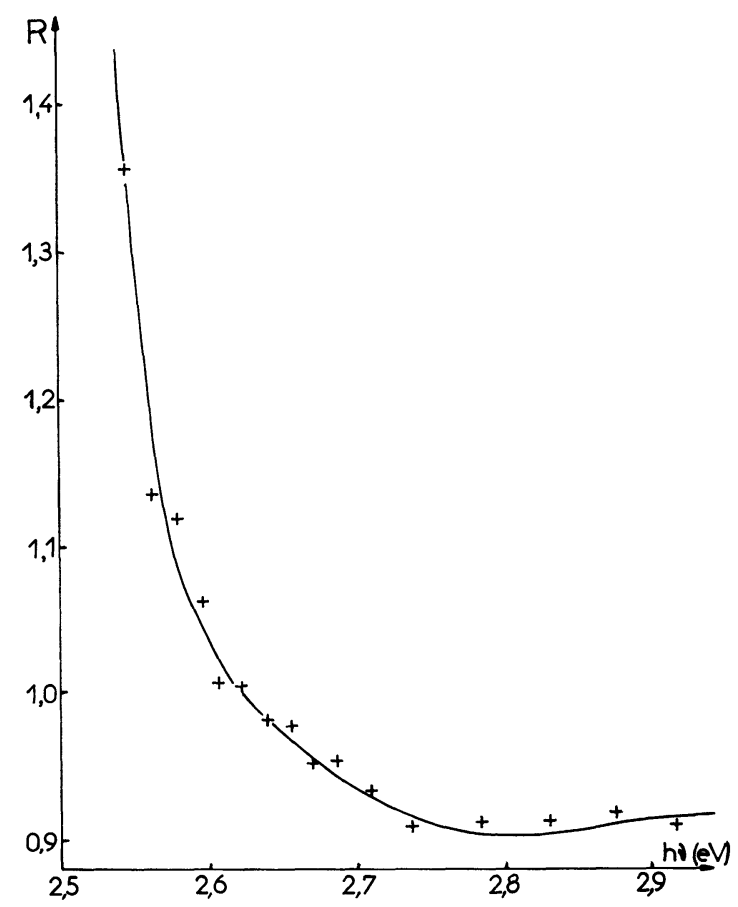

Frg. 1. - Action de la température sur le baryum. Graphe $R(h \nu)$ obtenu à partir des données expérimentales de Jamison et Cashman.

Il est évident que le rapport $\alpha^{\prime} / \alpha$ doit être indépendant de $\nu$. On a porté sur la figure 2 , en fonction de $h v$, les points correspondant à chacune des déterminations.

Alors que les points fournis par notre détermination (2 a) restent autour de la valeur prévue pour $\alpha^{\prime} / \alpha(0,906)$ en deçà des limites d'erreur $( \pm 0,04)$, les
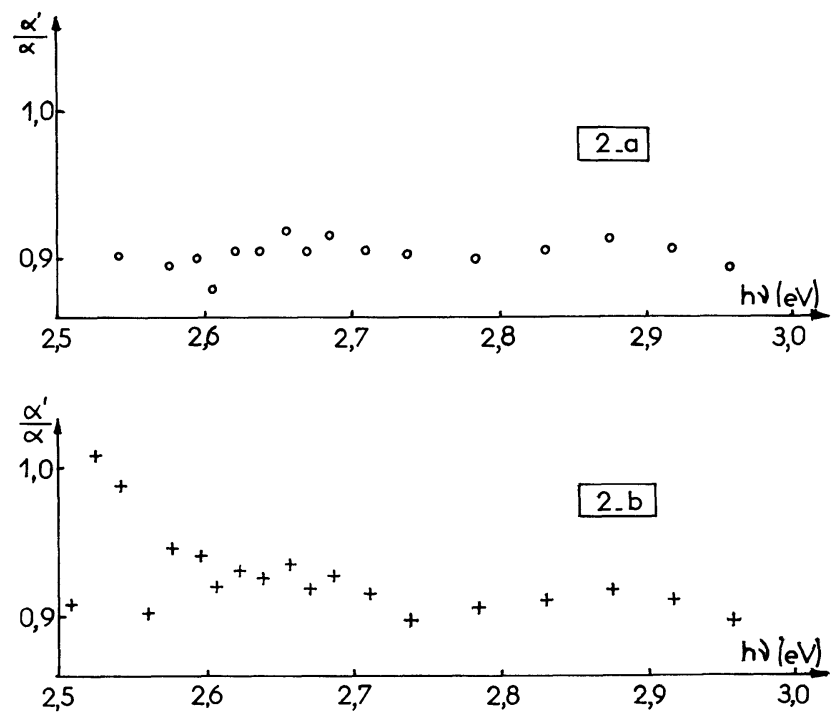

Fig. 2. - Action de la température sur le baryum. Variation du rapport $\alpha^{\prime} / \alpha$ en fonction de $h \nu: 2$ a) A partir de notre détermination; 2 b) A partir de la détermination de Jamison et Cashman. 
valeurs obtenues pour ce même rapport $(2 \mathrm{~b})$ à partir des résultats de Jamison et Cashman sont distribuées en fonction de la fréquence avec une dispersion de l'ordre de $10 \%$.

Il est évident que le fait de tenir compte, dans la méthode proposée, de l'invariance du rapport $\alpha^{\prime} / \alpha$ en fonction de la fréquence est un des éléments qui permet d'améliorer la précision des déterminations.

Conclusion. - Nous avons montré que la méthode proposée pour la détermination des grandeurs caractéristiques de l'effet photoélectrique présentait, du fait de sa conception, des avantages importants sur les méthodes classiques basées sur la même théorie.
On peut souligner que l'on dispose ainsi d'un moyen d'investigation, à la fois sensible et précis, des phénomènes de surface dont le rôle dans la Physique du Solide est particulièrement important.

Nous avons effectivement utilisé notre méthode pour étudier l'action de certains agents (température, champ électrique, induction magnétique) sur le potentiel de sortie et le coefficient de photo-ionisation des métaux. Les résultats obtenus feront l'objet d'une publication ultérieure. Les calculs nécessaires ont été effectués sur ordinateur Bull G.E. type CAB 500. Je remercie à ce propos mes collègues MM. R. Phan Tan Luu et L. Bouscasse, du Département de Chimie Organique, pour l'aide efficace qu'ils m'ont apportée.

\section{BIBLIOGRAPHIE}

[1] Fowlek (R. H.), Phys. Rev., 1931, 38, 45.

[2] Du BRIDGE (L. A.), New theories of the photoelectric effect, Hermann, Paris, 1935.

[3] LESSEN (L. VAN), cf. Simon et Suhrman, Der lichtelektrische Effect (und seine Anwendungen), Springer, Berlin, 1958, p. 28.
[4] Du Bridge (L. A.), Phys. Rev., 1932, 39, 108.

[5] Fisher (R. A.), Statistical methods for research workers, Oliver et Boyd, Edimbourg, 1949.

[6] Jamison (N. C.) et Cashman (R. J.), Phys. Rev., 1936, 50, 624 .

[7] MeEssen (A.), J. Physique Rad., 1959, 20, 519.

\section{INFORMATION}

Dans le cadre des JournéEs D'ÉLeGtronique de Toulouse, la Ville, l'Université, l'Industrie régionale organisent un Colloque International sur la Microélectronique, du 3 au 7 mars 1969, à Toulouse, France.

Programme. - I. Physique et chimie des matériaux de l'élegtronique (3 et 4 mars). Élaboration, contrôle, purification et dosage des solides présentant un intérêt par leurs propriétés électroniques ou magnétiques; Relations entre les propriétés électroniques ou magnétiques et les structures des solides; Techniques de fabrication en microélectronique.

Responsables scientifiques : MM. les Professeurs Fert et Montel.

II. RÉAlisations en microÉlegtronique. - II.1. Hyperfréquences (5 mars). - Générateurs (transistors, diodes tunnel, diodes à effet Gunn...); Circuits (microbandes, circuits intégrés...); Dispositifs actifs (détecteurs, mélangeurs...).

Responsables scientifiques : MM. les Professeurs Dupin et Lefeuvre.

II.2. Logique, Informatique ( 6 et 7 mars). - Présentation et étude des familles logiques intégrées (logiques saturées, logiques commutation de courant, dispositifs particuliers : mémoires, registres...); Technologie avancée en microélectronique (semiconducteurs : M.S.I., L.S.I., optoélectronique, dispositifs magnétiques...); Influence de la microélectronique sur l'architecture des systèmes informatiques (interconnexion, fiabilité, évolution des structures); Apport de l'informatique au développement de la microélectronique (conception logique, tests automatiques, réalisations automatiques des masques).

Responsables scientifiques : MM. Beaufils et Raynaud, Centre d'Informatique de Toulouse; M. le Professeur Cassignol, Directeur Général de Motorola à Toulouse.

Les personnes désireuses de présenter une communication sont priées d'en envoyer le titre et le résumé avant le 31 décembre 1968 au Secrétariat du Colloque : S. Lefeuvre, E.N.S.E.I.H.T., 2, rue Charles-Camichel, 31-Toulouse-01, Tél. : (61) 22.28.77, 22.54.20, Poste : 246. 